\title{
C-kit-negative Extragastrointestinal Stromal Tumor Originating in the Mesentery Misdiagnosed as an Ovarian Tumor before Surgery
}

\author{
Jongryeul Lim1, Myong Ki Baeg', Sangjeong Ahn², Man Ho Ha ${ }^{3}$, Sun-Hye Ko ${ }^{4}$, Hyuki Kwon ${ }^{1}$, Jaeho Han ${ }^{1}$ \\ Division of Gastroenterology, Department of Internal Medicine ${ }^{1}$, Departments of Pathology ${ }^{2}$ and Surgery ${ }^{3}$, Division of Hematology, \\ Department of Internal Medicine ${ }^{4}$, International St. Mary's Hospital, Catholic Kwandong University College of Medicine, Incheon, Korea
}

\begin{abstract}
Gastrointestinal stromal tumors (GISTs) are rare digestive system malignancies with extragastrointestinal stromal tumors (EGISTs) being even less. Diagnosing GISTs usually requires the identification of c-kit (CD117) expression by immunohistochemical staining. A 53-year-old woman complaining of dyspepsia was referred for the evaluation of a 1.5-cm extrinsic compression at the greater curvature of the proximal antrum. EUS revealed a multiseptated mass with positive Doppler findings. Abdominal CT showed that she harbored a large, $20-\mathrm{cm}$ mass in her abdominal cavity, most likely arising from the right ovary. Surgery revealed a hypervascular tumor arising from the mesentery and attached to the gastric lesser curvature. Pathological examination revealed negativity for c-kit, but positivity for the protein "Discovered on GIST-1" (DOG1), confirming the EGIST diagnosis. Herein, we report this rare case of a c-kit-negative EGIST originating in the mesentery, which was diagnosed based on staining for DOG1. (Korean J Helicobacter Up Gastrointest Res 2021;21:156-160)
\end{abstract}

Key Words: C-kit protein; DOG-1 protein; Gastrointestinal stromal tumor; Mesentery

\section{INTRODUCTION}

Gastrointestinal stromal tumors (GIST), which originate from the interstitial cells of Cajal, are rare, consisting of less than $1 \%$ of all primary gastrointestinal malignancies. ${ }^{1,2}$ The sites where GISTs mainly occur are the stomach (60 70\%), followed by the small intestine (20 25\%), colon and rectum $(5 \%)$, and esophagus $(<5 \%)^{2}$ In addition, GISTs found outside the gastrointestinal tract, so-called extragastrointestinal stromal tumor (EGIST), are even more rarer. ${ }^{3}$ In over 95\% of GISTs, CD117 are expressed with positive immunohistochemistry (IHC) staining for c-kit (CD117), and GISTs can also show expressions for CD34, actin, S-100 and desmin in descending order, at 70 90\%, 20 30\%, $8 \sim 10 \%$, 2 4\%, respectively. ${ }^{2,4}$ In case of the c-kit-negative GISTs, where those are not enough to confirm the diagnosis of GISTs, additional staining with Discovered on GIST-1 (DOG1) can be used. ${ }^{5}$ Here, we report a case of c-kit-negative EGIST arising in the mesentery, misdiagnosed

Received: December 1, 2020 Revised: December 31, 2020 Accepted: January 8, 2021

Corresponding author: Myong Ki Baeg

Department of Gastroenterology, International St. Mary's Hospital, 25 Simgok-ro 100beon-gil, Seo-gu, Incheon 22711, Korea

Tel: +82-32-290-3879, Fax: +82-32-290-3302, E-mail: baegmk@gmail.com as an ovarian tumor before surgical resection.

\section{CASE REPORT}

A 53-year-old woman, who was a nondrinker and a nonsmoker with no past history except for benign thyroid nodules diagnosed 2 years ago, was referred to the International St. Mary's Hospital, with symptoms of indigestion and weight loss by $2 \mathrm{~kg}$ for 1 month. Before the visit, upper gastrointestinal endoscopy was performed at a local clinic, which revealed a mass in the stomach, suspicious of a subepithelial tumor (SET). Her vital signs were within normal ranges and laboratory findings such as tumor markers were non-specific. Additionally endoscopy was done again in our hospital, and an almost $1.5 \mathrm{~cm}$ multinodular SET was observed on the greater curvature of the proximal antrum, and the mass showed a positive pillow sign (Fig. 1A). EUS revealed a $2.04 \times 2.83 \mathrm{~cm}$ irregular trapezoid anechoic mass with multiple septations, embedded in the submucosal layer (Fig. 1B). This mass was a multiseptated cystic lesion with mixed Doppler ultrasound signals, which was only a small portion of a huge solid mass extending outwards from the gastric wall and it was hard to discern how large it was (Fig. 1C).

Copyright $\odot 2021$ Korean College of Helicobacter and Upper Gastrointestinal Research

(a) The Korean Journal of Helicobacter and Upper Gastrointestinal Research is an Open-Access Journal. All articles are distributed under the terms of the Creative Commons Attribution Non-Commercial License (http:// creativecommons.org/licenses/by-nc/4.0) which permits unrestricted non-commercial use, distribution, and reproduction in any medium, provided the original work is properly cited. 
Contrast-enhanced stomach cancer preoperative three-dimensional CT images were taken, and they displayed a roughly $21 \mathrm{~cm}$-sized mixed hypervascular mass, attached to the stomach lower body and antrum and extending to her right adnexa (Fig. 2A). There was no evidence of distant metastasis nor lymphadenopathy in the abdominopelvic cavity.

Based on the results of the CT scan, the patient was referred to the department of gynecology as the lesion was considered a tumor that developed from the right ovary rather than the gastrointestinal system. Transvaginal ultrasound was done, which showed an $8.36 \times 4.59 \mathrm{~cm}$ right ovarian mass with irregular margins and mixed echogenicity (Fig. 2B). She also underwent 18F-fluorodeoxyglucose (18F-FDG) PET, which revealed a huge mass in the abdominopelvic cavity, showing increased FDG uptake at the solid portion of the lesion, suggestive of malignancy originating from the right ovary. No other discernible abnormal FDG uptake to suggest metastasis was observed on the 18F-FDG PET images (Fig. 2C).

Surgical resection was decided by the gynecologist with the impression of malignant ovarian tumor. However, laparoscopy revealed that there was no connection between the mass and the right ovary (Fig. 3A). The gynecologist contacted the gastrointestinal surgeon and a midline incision was made for an open laparotomy to remove the mass. The tumor was approximately $20 \mathrm{~cm}$, soft, friable, well-marginated and noninvasive, and blood supply into the posterior portion of the mass from the greater omentum was observed (Fig. 3C). As the only connection of the mass to the adjacent organs was the greater curvature of stomach lower body (Fig. 3B), in addition to the mesenteric tumor excision, gastric wedge resection was also performed for a complete gross excision.
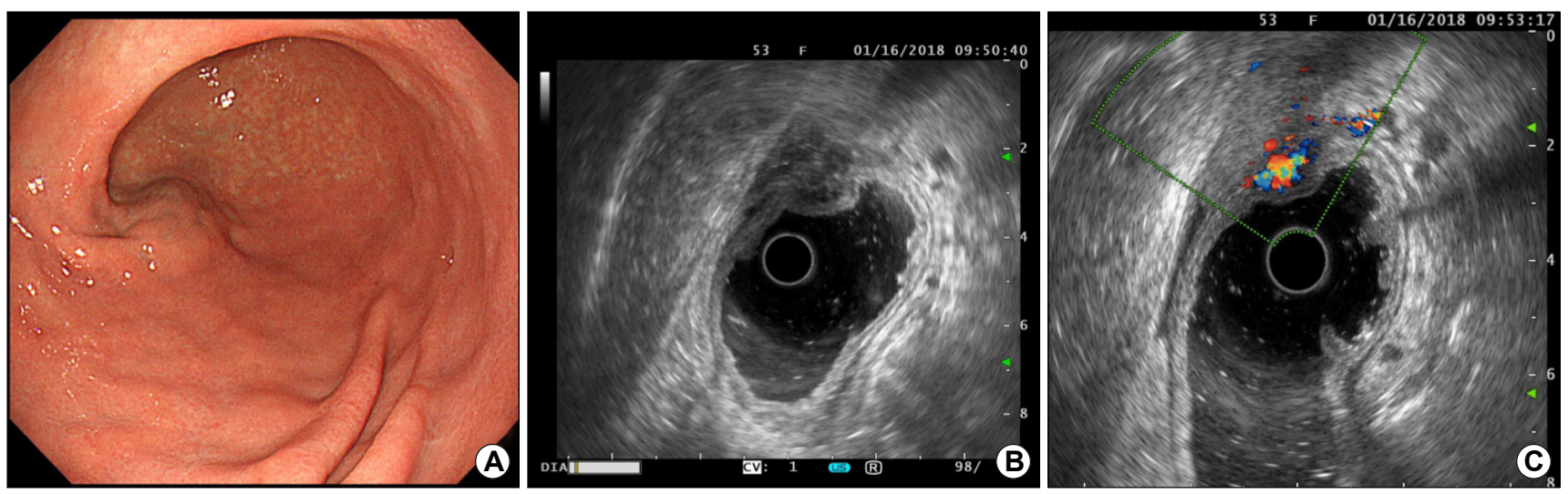

Fig. 1. (A) A 1.5-cm soft mass is seen on the greater curvature of the antrum. (B) EUS shows an irregular anechoic lesion with multiple septations embedded in the submucosal layer. (C) Mixed doppler ultrasound signals are seen within the mass.
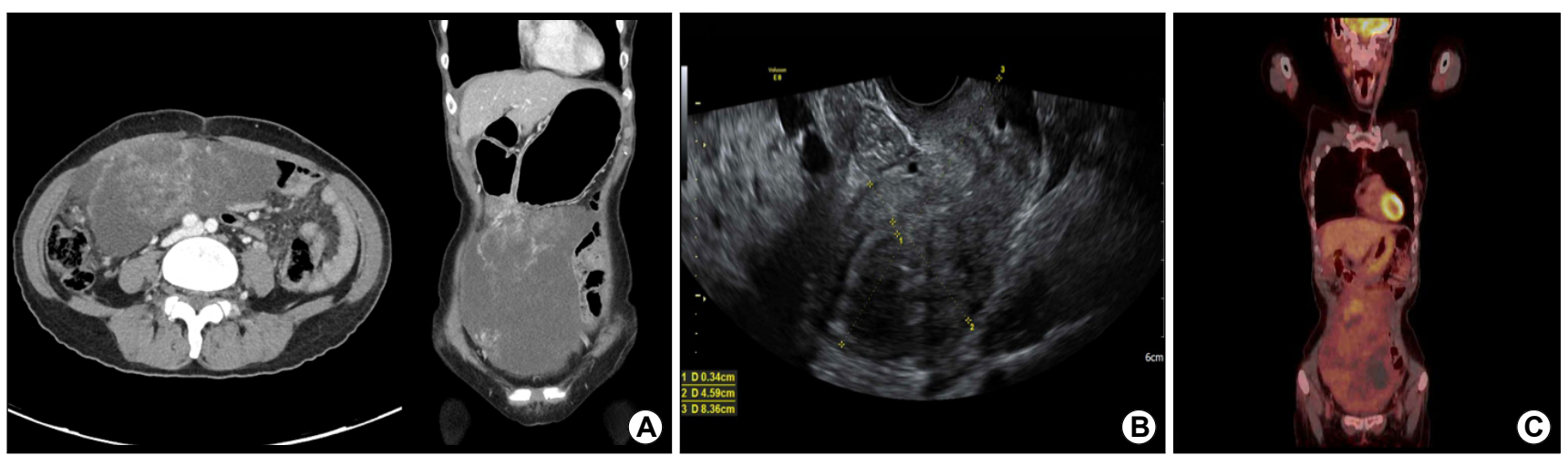

Fig. 2. (A) A 21-cm mass is observed to be attached to the lower body of the stomach and antrum and extending to the right adnexa. (B) A right ovarian mass with mixed echogenicity. (C) Increased fluorodeoxyglucose uptake is observed in the solid portion of the mass. 

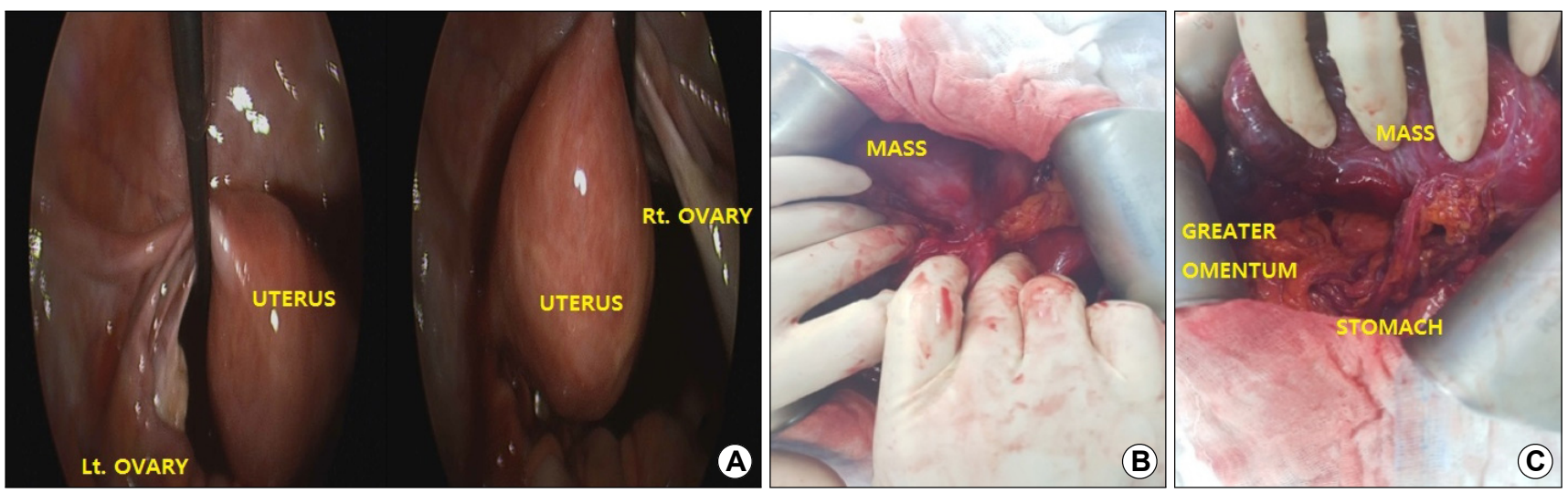

Fig. 3. (A) No connection was noted between the lesion and right ovary. (B) Only adhesion of the mass to the greater curvature of lower body of the stomach is observed. (C) The blood supply into the mass from the greater omentum is observed. Lt., left; Rt., right.
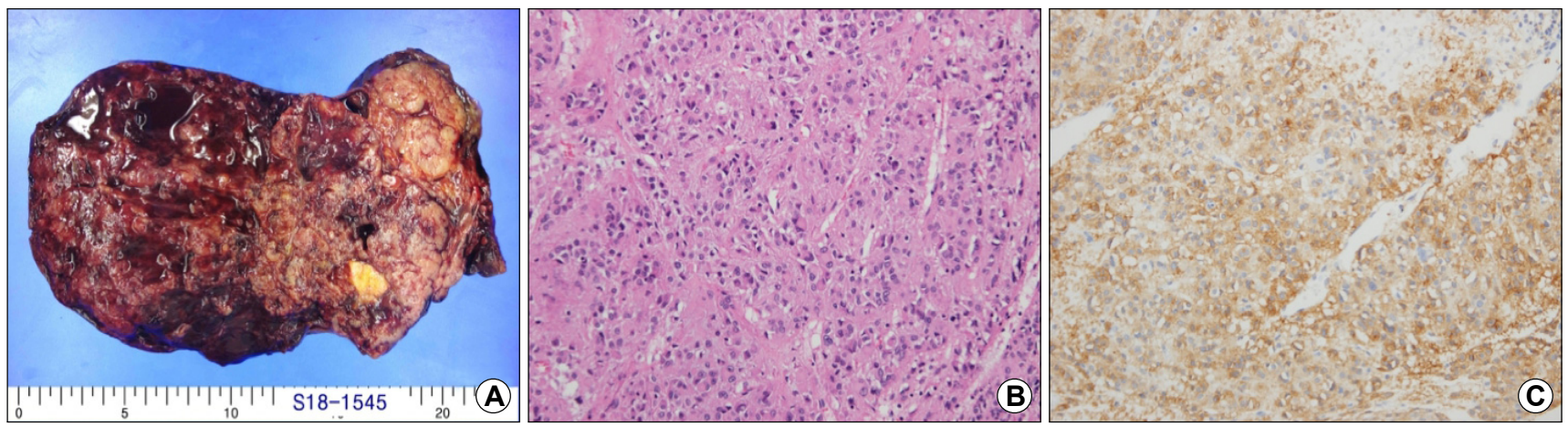

Fig. 4. (A) A gross examination shows a large, grayish tan, solid, and partly degenerative cystic mass. (B) Microscopically, tumor cells show distinctive epithelioid features $(\mathrm{H} \& \mathrm{E}, \times 400)$. (C) Immunohistochemistry for Discovered on GIST-1 reveals strong positivity (immunohistochemical staining, $\times 400)$.

Grossly, an encapsulated huge brown mass, $20.0 \times 14.0 \times 5.0$ $\mathrm{cm}$ in size, was found with partial adhesion to the gastric wall and omental tissue. The mass showed a relatively well-defined gray tan multinodular solid and partly cystic appearance with exuberant hemorrhagic features (Fig. 4A). Moreover, a focal yellowish necrotic area $(<5 \%)$ was noted. Microscopically, tumor cells were composed of sheets of distinctive epithelioid cells with round nucleoli, small nucleoli, and abundant palely basophilic or eosinophilic cytoplasm (Fig. 4B). Pleomorphic and multinucleated giant cells were occasionally noted, which is rare in GIST, but mostly seen in epithelioid tumors. The tumor contained abundant myxoid stroma and dilated vasculature. Mitosis showed a low rate ( $<1 / 50$ high power fields). On immunohistochemical exams, the tumor was positive for DOG1 (Fig. 4C) and CD34, and negative for c-kit (CD117), smooth muscle actin (SMA) and S-100. Based on the morphologic and immunohistochemical results, the patient was diagnosed with a malignant EGIST of the mesentery in a high risk category, according to the National Institutes of Health (NIH)-Fletcher criteria. ${ }^{6}$

After the operation, she was discharged on post-operative day 19, and adjuvant treatment was planned. One month later, she began to take imatinib mesylate, $400 \mathrm{mg}$ once daily. At 1-year post-surgery, there was no evidence of recurrence on esophagogastroduodenoscopy and abdominopelvic CT.

\section{DISCUSSION}

GISTs are rare tumors that make up a small portion of gastrointestinal neoplasms. Moreover, EGISTs are even rarer as they account for only 10\% of all stromal tumors. ${ }^{7}$ Although the main sites where EGISTs occur are known to be the mesentery, omentum, and retroperitoneum, they 
also have been reported to be found in other organs including the bladder and pancreas, just to name a few. ${ }^{8-10}$

In GISTs more than 95\% have c-kit (CD117) expression with diffuse cytoplasmic staining patterns. In $75 \%$ of the cases, intense staining is seen. In addition, about $70 \%$ of GISTs are positive for CD34, 20 30\% are positive for SMA, 10\% are positive for S100 protein, and less than 5\% are positive for desmin. ${ }^{2}$ Meanwhile, nearly 5\% of GISTs with typical morphological features are negative for CD117.,11 Those with negative stains or weak staining in less than $10 \%$ of the tumor are considered as such. For c-kit-negative GISTs, the almost pathognomonic IHC marker DOG1, corresponding to the potassium transporter anoctamin-1, can optionally be included in the initial IHC panel and is highly recommended, since over a third of them show DOG1 expression. ${ }^{12}$ On top of that, there were some studies reporting that DOG1 was more useful than CD117 because it had much higher sensitivity especially in the cases of c-kit-negative GISTs. ${ }^{12,13}$ Therefore, doctors can confirm a diagnosis of GISTs with the IHC expression of CD117 and/or DOG1.

When it comes to the relation between the IHC expression for CD117 or DOG1 and the prognosis of GISTs, several studies have been performed, but it still appears to be a highly controversial issue. For CD117, a recent study said that the prognoses and predictive factors for prognosis of c-kit-negative GISTs remain unclear. ${ }^{14}$ Some researchers have claimed that DOG1 overexpression was associated with adverse prognosis, suggesting that immunostaining index of DOG1 should be utilized as a routine test when diagnosing GIST, as it might be valuable as a potential prognostic tool and a target for novel therapies. ${ }^{15}$ However, other studies have reported that CD117 and DOG1 are not an indicator of prognosis, although they proved to be useful as diagnostic tools. ${ }^{16}$ Thus, further investigation is required to clarify if the IHC expressions for such markers are related to GIST prognosis.

There are several risk assessment criteria with regard to GIST recurrence after surgical excision. When considering the fact that all the criteria has one thing in common, which is "the larger a GIST is or the higher its mitotic index is, the higher recurrence risk the patient has', the patient mentioned above falls within the high risk group. As the size of the tumor was very large, up to 20 $\mathrm{cm}$, according to NH-Fletcher criteria, the recurrence risk was high regardless of its mitotic index. ${ }^{6}$

Recently, many studies have reported that adjuvant therapy with imatinib, a competitive inhibitor of tyrosine kinases, significantly decreased the recurrence rate in high-risk patients. For example, in a 2016 follow-up analysis with median follow up of 90 months, the 5 year relapse free survival was $71 \%$ for 3 years of imatinib vs. $52 \%$ for 1 year of imatinib, and the 5 year overall survival was $92 \%$ vs. $85 \%$ respectively (hazard ratio, $0.60 ; 95 \% \mathrm{CI}$, 0.37 to $0.97 ; P=0.036) .{ }^{17}$ Since KIT or PDGFRA mutation can exist in c-kit-negative GISTs as well as c-kit-positive ones, adjuvant imatinib accompanying surgery has also been considered an effective treatment for the patients with c-kit-negative GISTs. ${ }^{18}$ Accordingly, the administration of imatinib for this patient as an adjuvant treatment is thought to be appropriate and reasonable.

To sum up, this case is unique in that the tumor was a c-kit-negative EGIST originating in the mesentery, which had been mistaken for ovarian cancer prior to surgery. Through this experience, we want to report the case in order to emphasize that DOG1 expression should be checked for patients who are clinically suspected of having a c-kit-negative EGIST even if the mass is not located in the gastrointestinal tract.

\section{CONFLICT OF INTEREST}

No potential conflicts of interest relevant to this article were reported.

\section{ORCID}

$\begin{array}{ll}\text { Jongryeul Lim } & \text { (D)https://orcid.org/0000-0003-3020-8323 } \\ \text { Myong Ki Baeg } & \text { (D https://orcid.org/0000-0002-4807-2447 } \\ \text { Sangjeong Ahn } & \text { (D) https://orcid.org/0000-0003-4338-3235 } \\ \text { Man Ho Ha } & \text { (D https://orcid.org/0000-0002-4815-0820 } \\ \text { Sun-Hye Ko } & \text { (D) https://orcid.org/0000-0003-3387-3986 } \\ \text { Hyuki Kwon } & \text { (D) https://orcid.org/0000-0002-3801-6273 } \\ \text { Jaeho Han } & \text { (D) https://orcid.org/0000-0001-9977-1873 }\end{array}$




\section{REFERENCES}

1. Cajal SR. About the ganglia and nerve plexus of the intestine. C R Seances Soc Biol Fil 1893;45:217-223.

2. Miettinen M, Lasota J. Gastrointestinal stromal tumors--definition, clinical, histological, immunohistochemical, and molecular genetic features and differential diagnosis. Virchows Arch 2001;438:1-12.

3. Erlandson RA, Klimstra DS, Woodruff JM. Subclassification of gastrointestinal stromal tumors based on evaluation by electron microscopy and immunohistochemistry. Ultrastruct Pathol 1996; 20:373-393.

4. Corless CL, Heinrich MC. Molecular pathobiology of gastrointestinal stromal sarcomas. Annu Rev Pathol 2008;3:557-586.

5. Medeiros F, Corless CL, Duensing A, et al. KIT-negative gastrointestinal stromal tumors: proof of concept and therapeutic implications. Am J Surg Pathol 2004;28:889-894.

6. Fletcher CD, Berman JJ, Corless C, et al. Diagnosis of gastrointestinal stromal tumors: a consensus approach. Hum Pathol 2002;33:459-465.

7. Miettinen M, Monihan JM, Sarlomo-Rikala M, et al. Gastrointestinal stromal tumors/smooth muscle tumors (GISTs) primary in the omentum and mesentery: clinicopathologic and immunohistochemical study of 26 cases. Am J Surg Pathol 1999; 23:1109-18.

8. Pidhorecky I, Cheney RT, Kraybill WG, Gibbs JF. Gastrointestinal stromal tumors: current diagnosis, biologic behavior, and management. Ann Surg Oncol 2000;7:705-712.

9. Krokowski M, Jocham D, Choi H, Feller AC, Horny HP. Malignant extragastrointestinal stromal tumor of bladder. J Urol
2003;169:1790-1791.

10. Vij M, Agrawal V, Pandey R. Malignant extra-gastrointestinal stromal tumor of the pancreas. A case report and review of literature. JOP 2011;12:200-204.

11. Debiec-Rychter M, Wasag B, Stul M, et al. Gastrointestinal stromal tumours (GISTs) negative for KIT (CD117 antigen) immunoreactivity. J Pathol 2004;202:430-438.

12. Liegl B, Hornick JL, Corless CL, Fletcher CD. Monoclonal antibody DOG1.1 shows higher sensitivity than KIT in the diagnosis of gastrointestinal stromal tumors, including unusual subtypes. Am J Surg Pathol 2009;33:437-446.

13. Miettinen M, Wang ZF, Lasota J. DOG1 antibody in the differential diagnosis of gastrointestinal stromal tumors: a study of 1840 cases. Am J Surg Pathol 2009;33:1401-1408.

14. Seo HS, Hyeon JY, Shin OR, Lee HH. C-kit-negative gastrointestinal stromal tumor in the stomach. J Gastric Cancer 2015;15:290-294.

15. Şahin S, Ekinci Ö, Seçkin S, Dursun A. The diagnostic and prognostic utility of DOG1 expression on gastrointestinal stromal tumors. Turk Patoloji Derg 2017;33:1-8.

16. Kövecsi A, Jung I, Szentirmay Z, et al. PKC $\theta$ utility in diagnosing c-KIT/DOG-1 double negative gastrointestinal stromal tumors. Oncotarget 2017;8:55950-55957.

17. Joensuu H, Eriksson M, Sundby Hall K, et al. Adjuvant imatinib for high-risk GI stromal tumor: analysis of a randomized trial. J Clin Oncol 2016;34:244-250.

18. West RB, Corless CL, Chen X, et al. The novel marker, DOG1, is expressed ubiquitously in gastrointestinal stromal tumors irrespective of KIT or PDGFRA mutation status. Am J Pathol 2004; 165:107-113. 